\title{
Analysis of crack-tip plastic zone in a Compact Tensile Shear (CTS) Specimen
}

\author{
C. M. Sharanaprabhu \\ Department of Mechanical Engineering, Bapuji Institute of Engineering \& Technology, Davanagere 577004, India
}

\author{
Shashidhar K. Kudari \\ Research Center, Department of Mechanical Engineering SDM, College of Engineering and Technology, \\ Dharwad-580002, India \\ s.kudari@rediffmail.com
}

\begin{abstract}
The minimum plastic zone radius (MPZR) criterion is one of the recent criterions to estimate crack initiation direction under mixed mode loading. The MPZR theory is based on the theoretical computations of plastic zone size (PZS). In this investigation, the shape and size of crack-tip plastic zones have been estimated by analytical and the elastic finite element computations in a Compact Tensile Shear (CTS) specimen under mixed mode (I/II) loading according to von Mises yield criteria. The theoretical and the finite element analysis results are compared to analyze the minimum plastic zone radius (MPZR) criterion for crack-initiation angle with reference to the loading angle and stress intensity factor.
\end{abstract}

KEYwORDS. Plastic zone; Mixed mode I/II; Minimum plastic zone radius; Finite element analysis.

\section{INTRODUCTION}

$\mathrm{P}$ rediction of crack initiation and orientation with its propagation path under mixed-mode loading is desirable for life prediction of engineering materials [1-3]. In mixed mode fracture, it is known that the crack initiation angle depends on the loading angle [4], and the loading angle alters the shape and size of the crack-tip plastic zone. Several investigators [5-7] have proposed fracture criteria for the prediction of the crack-initiation angle based on the analytical estimates of crack-tip plastic zone size in mixed mode fracture. The analytical estimates were obtained by Linear Elastic Fracture Mechanics (LEFM) approach. Recently, Bian and Kim [4] and Khan and Khraisheh [7] have proposed a minimum plastic zone radius (MPZR) theory for crack initiation angle in mixed mode monotonic loading. MPZR theory states that the crack initiates in the direction where the radius of plastic zone takes either a local or global minimum depending on the loading direction. It is well known that plastic zone size (PZS) depends on the type of the fracture specimen [8]. Hence, this kind of study needs detailed information about the crack-tip plastic zone shape and size in a fracture specimen estimated by numerical method such as the finite element method. Benrahou et al. [9] have estimated the plastic zone under mixed mode loading by the finite element method, but the details of the mixed mode plastic zone analysis are missing in their investigation. Sharanaprabhu and Kudari [10] have studied size and shape of plastic zone under mixed mode loading condition using finite element analysis. The authors have demonstrated that MPZR varies linearly with respect effective stress intensity factor and is independent of loading angle. Hence, the objective of the present work is to validate the finite element results with the theoretical results to analyze the minimum plastic zone radius criterion for crack initiation angle with reference to the loading angle and stress intensity factor in linear elastic fracture mechanics (LEFM) regime for plane stress condition. 


\section{ANALYTICAL ESTIMATION OF CRACK INITIATION ANGLE}

or a singular elastic field of an infinite plate with a crack, the stress near the crack tip expressed in terms of Cartesian co-ordinates $(x, y)$ shown in Fig.1 are given as [4].

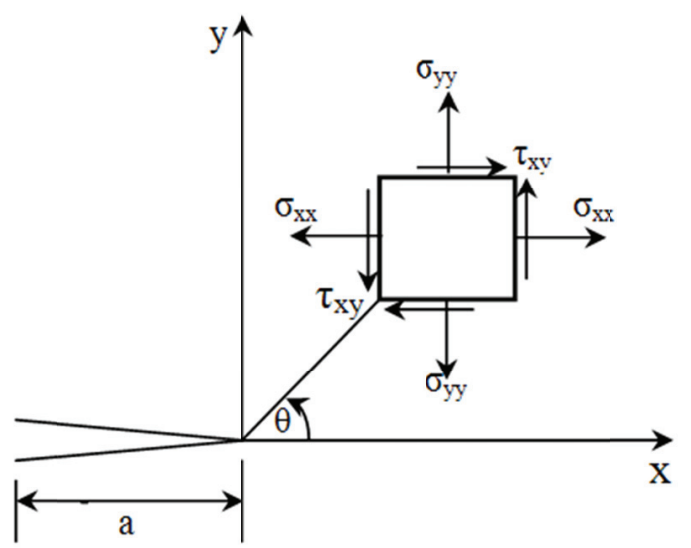

Figure 1: Crack tip stresses expressed in Cartesian co-ordinates.

$$
\begin{aligned}
& \sigma_{x x}=\frac{1}{\sqrt{2 r}}\left[K_{I} \cos \frac{\theta}{2}\left(1-\sin \frac{\theta}{2} \sin \frac{3 \theta}{2}\right)-K_{I I} \sin \frac{\theta}{2}\left(2+\cos \frac{\theta}{2} \cos \frac{3 \theta}{2}\right)\right] \\
& \sigma_{y y}=\frac{1}{\sqrt{2 r}}\left[K_{I I} \cos \frac{\theta}{2}\left(1+\sin \frac{\theta}{2} \sin \frac{3 \theta}{2}\right)+K_{I I} \sin \frac{\theta}{2} \cos \frac{\theta}{2} \cos \frac{3 \theta}{2}\right] \\
& \tau_{x y}=\frac{1}{\sqrt{2 r}}\left[K_{I} \sin \frac{\theta}{2} \cos \frac{\theta}{2} \cos \frac{3 \theta}{2}+K_{I I} \cos \frac{\theta}{2}\left(1-\sin \frac{\theta}{2} \sin \frac{3 \theta}{2}\right)\right] \\
& \sigma_{z z}=v\left(\sigma_{x x}+\sigma_{y y}\right)=\frac{2 v}{\sqrt{2 r}}\left[\mathrm{~K}_{I} \cos \frac{\theta}{2}-K_{I I} \sin \frac{\theta}{2}\right] \quad \text { Plane Strain } \\
& \sigma_{z z}=0 \quad \text { Plane stress }
\end{aligned}
$$

The stress intensity factor for CTS specimen of interest is given by [11].

$$
\begin{gathered}
K_{I}=\frac{p \sqrt{\pi b}}{w t} \frac{\cos \beta}{\left(1-\frac{b}{w}\right)} \sqrt{\frac{0.26+2.65\left(\frac{b}{(w-b)}\right)}{1+0.55\left(\frac{b}{w-b}\right)-0.08\left(\frac{b}{w-b}\right)^{2}}} \\
K_{I I}=\frac{p \sqrt{\pi b}}{w t} \frac{\sin \beta}{\left(1-\frac{b}{w}\right)} \sqrt{\frac{-0.23+1.40\left(\frac{b}{(w-b)}\right)}{1-0.67\left(\frac{b}{w-b}\right)-2.08\left(\frac{b}{w-b}\right)^{2}}}
\end{gathered}
$$


where $p$ is the applied force, $b$ is the crack depth of the specimen, $w$ is the width of the specimen, $t$ is the thickness of the specimen and $\beta$ is the loading angle.

\section{MINIMUM PLASTIC ZONE RADIUS CRITERION}

I

$\mathrm{t}$ is assumed in this study that the direction of crack initiation coincides with the direction of minimum plastic zone radius evaluated from the von Mises yield criterion. The crack initiation can be determined by minimizing $r$ [4]:

$$
\left(\frac{\partial r}{\partial \theta}\right)_{\theta=\theta_{o}}=0 \quad\left(\frac{\partial^{2} r}{\partial \theta^{2}}\right)_{\theta=\theta_{O}}>0
$$

Where $r$ is the radius of plastic zone. The von Mises yield criteria for the three dimensional object can be written in the following form.

$$
\left(\sigma_{x x}-\sigma_{y y}\right)^{2}+\left(\sigma_{y y}-\sigma_{z z}\right)^{2}+\left(\sigma_{z z}-\sigma_{x x}\right)^{2}+6\left(\tau_{x y}^{2}+\tau_{y z}^{2}+\tau_{z x}^{2}\right)=2 \sigma_{y}^{2}
$$

where $\sigma_{\mathrm{y}}$ is the yield stress in uniaxial tension. As an approximate determination of the plastic zone, one can substitute the singular stress field of equations 1 to 3 in the above yield criterion and solve for the plastic zone radius $r$. It can be expressed in the following form.

$$
\begin{aligned}
& r\left(K_{I}, K_{I I}, \theta\right)=\frac{1}{4 \sigma_{y}^{2}}\left[K_{I}^{2}\left\{\frac{3}{4}(1-\cos 2 \theta)+2 \cos ^{2} \frac{\theta}{2}\right\}+K_{I I}^{2}\left\{\frac{15}{4}+2 \sin ^{2} \frac{\theta}{2}+\frac{9}{4} \cos 2 \theta\right\}\right. \\
& \left.+K_{I} K_{I I}\left\{\frac{52}{16} \sin 2 \theta+\frac{6}{16} \sin 3 \theta-2 \sin \theta\right\}\right]
\end{aligned}
$$

The crack initiation angle is given by Eq. 8 , the relative minimum must have positive circumferential stress.

\section{FINITE ELEMENT ANALYSIS}

7 he general-purpose finite element (FE) code ABAQUS is used in this study. A Compact Tensile Shear (CTS) specimen [10] under mixed mode loading has been considered in the present study. This kind of specimen is also referred to as the Compact Mixed Mode (CMM) specimen [4]. The specimen geometry used in the analysis is shown in Fig.2, the dimensions of the specimen considered in the analysis are similar to the one used in the work of Borrego et al. [12]. The loading of the specimen is applied at various angles ( $\beta$ ), $0^{\circ}$ (pure Mode-II), $18^{\circ}, 36^{\circ}, 54^{\circ}, 72^{\circ}$ and $90^{\circ}$ (pure Mode -I) to study the plastic deformation ahead of the crack-tip. The load is applied at various angles $\beta$ using a loading jig [10] along the six holes as shown in Fig. 3. In the present FE analysis the specimen loading at various angles $(\beta)$ is ascertained by applying uniaxial point loads $F_{1}$ to $F_{6}$ as shown in the Fig. 4 and estimated by Eqns. 11-13 as given below, in the similar manner to that demonstrated by Richard [11].

$$
\begin{aligned}
& F_{1}=F_{6}=F\left(\frac{1}{2} \cos \beta+\frac{c}{b} \sin \beta\right) \\
& F_{2}=F_{5}=F \sin \beta \\
& F_{3}=F_{4}=F\left(\frac{1}{2} \cos \beta-\frac{c}{b} \sin \beta\right)
\end{aligned}
$$

A series of elastic finite element calculations have been made on the CTS specimen (Fig. 2) considering the full specimen geometry due to the lack of loading symmetry. A typical two-dimensional FE mesh used in the analysis is shown in Fig. 4. The loading and displacement boundary conditions used in this analysis are similar to the one used in the work of Borrego et al. [12] and are clearly shown in Fig.4. Two-dimensional elastic FE calculations were performed using eight noded iso- 
parametric quadrilateral elements considering plane stress condition. The number of elements used in the FE analysis was 2844. The maximum load applied is $10 \mathrm{kN}$ and the corresponding applied stress is $62 \mathrm{MPa}$, which is approximately one third of yield stress to keep the analysis domain approximately under LEFM. In these calculations, the material behavior has been considered to be linear elastic type pertaining to interstitial free steel (IF) possessing the yield strength $\left(\sigma_{\mathrm{y}}\right)$ of 155 $\mathrm{MPa}$, Poisson's ratio (v) of 0.3 and elastic modulus (E) of $197 \mathrm{GPa}$ [8].

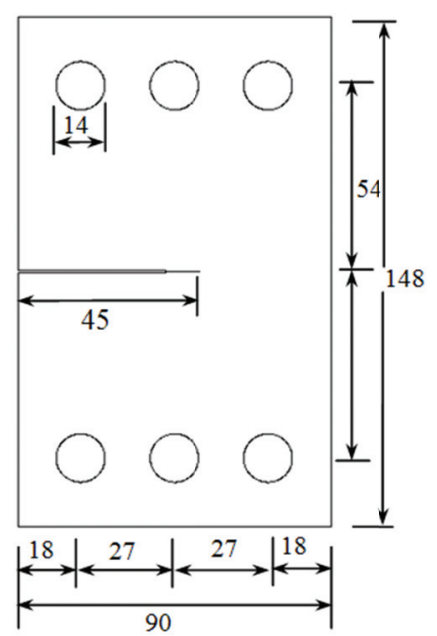

Figure 2: Specimen configuration used in the analysis (all dimensions in $\mathrm{mm}$ ), thickness $=3 \mathrm{~mm}$.

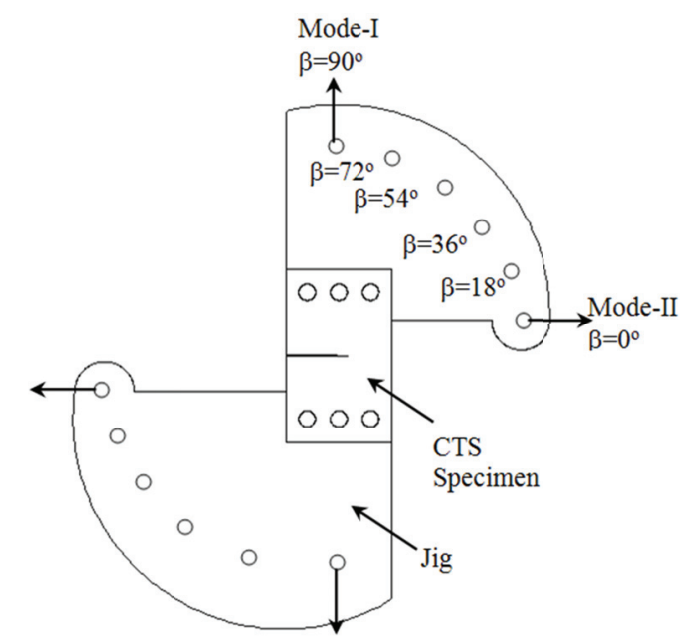

Figure 3: Loading Jig.

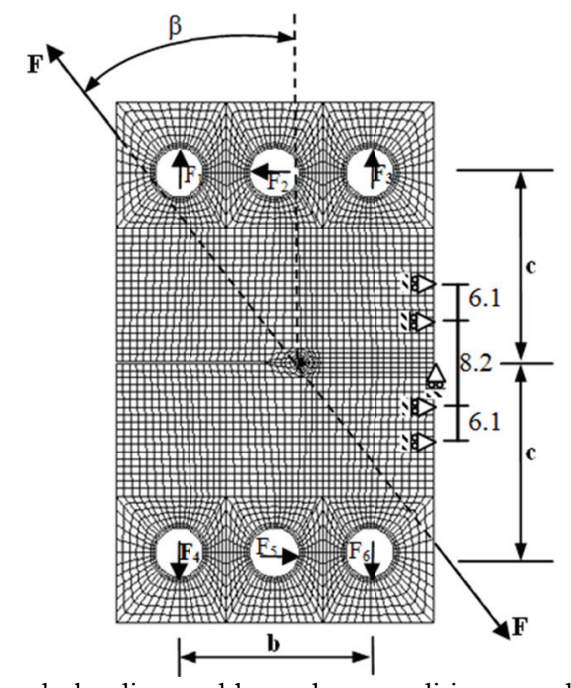

Figure 4: FE mesh, loading and boundary conditions used in FE analysis.

\section{RESULTS AND DISCUSSION}

ifferent load steps were applied to the specimen to estimate the stress intensity factor and to study the plastic zone shape and size ahead of the crack-tip. The stress intensity factors in mixed mode loading $\left(K_{I}\right.$ and $\left.K_{I I}\right)$ were computed for various load steps and loading angles $(\beta)$ using the ABAQUS post processor. The failure locus under mixed mode loading i.e variation of $\mathrm{K}_{\mathrm{II}}$ vs. $\mathrm{K}_{\mathrm{I}}$ for various loading is depicted in Fig.5. This figure indicates that for the similar applied load, for a loading angle below $70^{\circ}$ the stress intensity factor in mode-I is more than that of mode-II. This nature of variation of $K_{I I}$ vs. $K_{I}$ is in good agreement with the results shown by Benrahou et al. [9] and Kudari and Sharanaprabhu [13]. The magnitudes of $K_{I}$ and $K_{I I}$ have also been computed by analytical formulations cited in the above Eqns. (6) and (7). The estimated theoretical values of stress intensity factors have been superimposed in Fig. 5 typically for load $4 \mathrm{kN}$ and $10 \mathrm{kN}$. This plot indicates that there exists some discrepancy in the estimation of stress intensity factors by 
analytical formulation and present FE results. It is found that there is $10.5 \%$ and $2.4 \%$ error in estimation of $K_{I}$ and $K_{I I}$ respectively. This discrepancy in estimated magnitudes of stress intensity factor attributed to varied loading condition in $\mathrm{FE}$ analysis through loading Jig. The effective stress intensity factors $\left(\mathrm{K}_{\mathrm{eff}}\right)$ under mixed mode loading have been computed using the relation [9]:

$$
\mathrm{K}_{\mathrm{eff}}=\sqrt{\mathrm{K}_{\mathrm{I}}^{2}+\mathrm{K}_{\mathrm{II}}^{2}}
$$

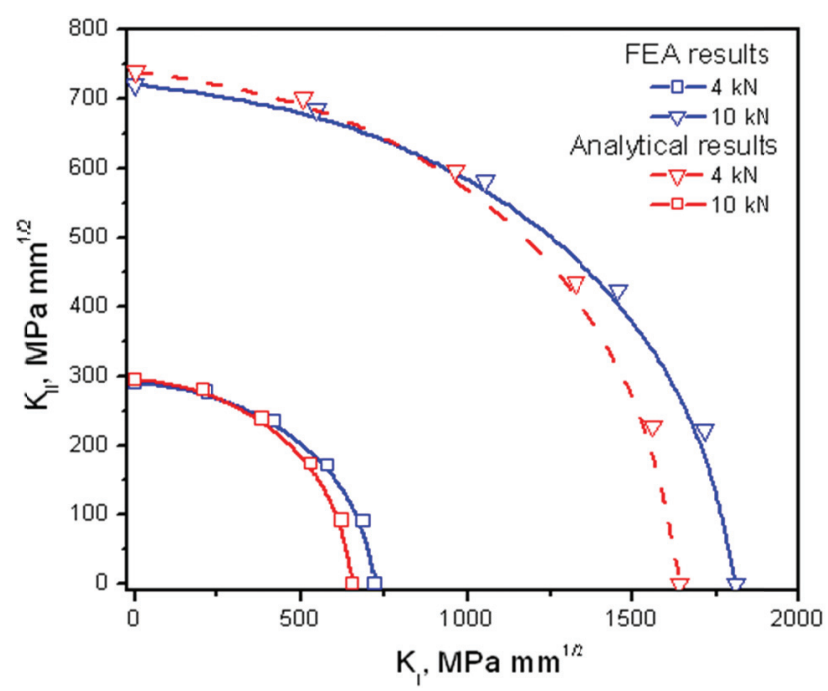

Figure 5: Variation of $\mathrm{K}_{\text {II }} v$ s. $\mathrm{K}_{\mathrm{I}}$ for different applied loads.

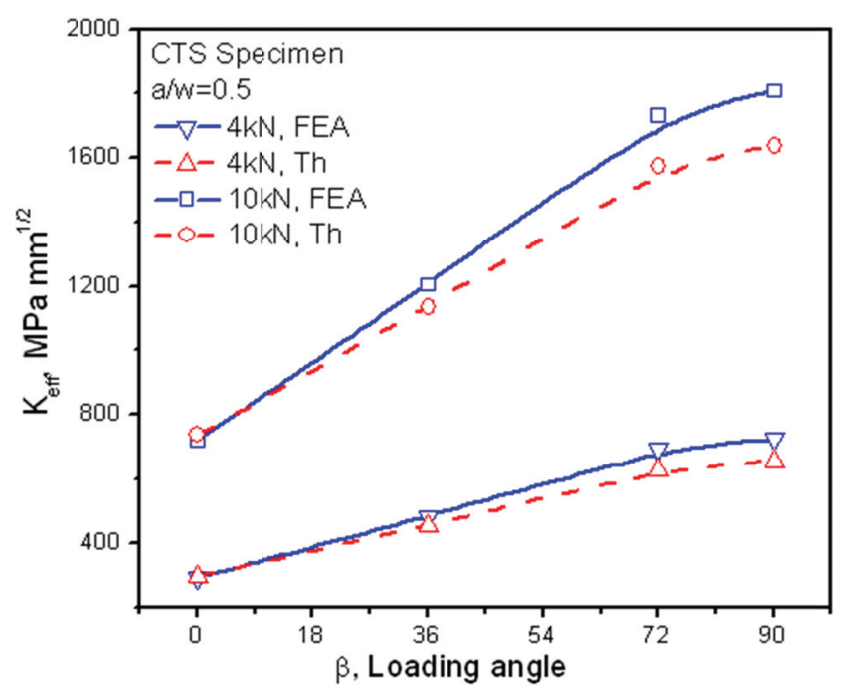

Figure 6: Variation of $K_{\text {eff }} v$ s. $\beta$ for different applied loads.

The computed magnitudes of $K_{\text {eff }}$ estimated by analytical and the finite element analyses are plotted against loading angle $(\beta)$ for various applied loads in Fig.6. The nature of variation of $K_{\text {eff }} v$ s. $\beta$ (Fig.6) is in good agreement with similar earlier results $[9,13]$.

The plastic zone shapes obtained by the Eq. 10 is shown in Fig.7. This figure indicates that the shape of plastic zone is dependent on loading angle. The shape of the plastic zone ahead of a crack-tip by FEA has been ascertained by plotting iso-contours of the effective stress, which causes yielding according to von Mises criterion [14]. Since, in this study elastic constitutive model is used for the material, contour plotting of the von Mises stress corresponds to the plastic zone shape. This is different from the actual plastic zone that is affected by stress redistribution during plastic flow. However, for the purposes of the present study the consideration is focused on the plastic zone, due to the considerably greater ease of analysis. The sequential development of crack-tip plastic zone for various applied loads and typically for loading angles $\beta=36^{\circ}$ (Mixed mode-I and II) is shown in Fig. 8. The plastic zone contours in Fig. 8 are obtained by superimposing the plastic zone contour obtained in each load step. For simplicity the displacement scaling of the specimens shown in Fig. 8 are set to zero.

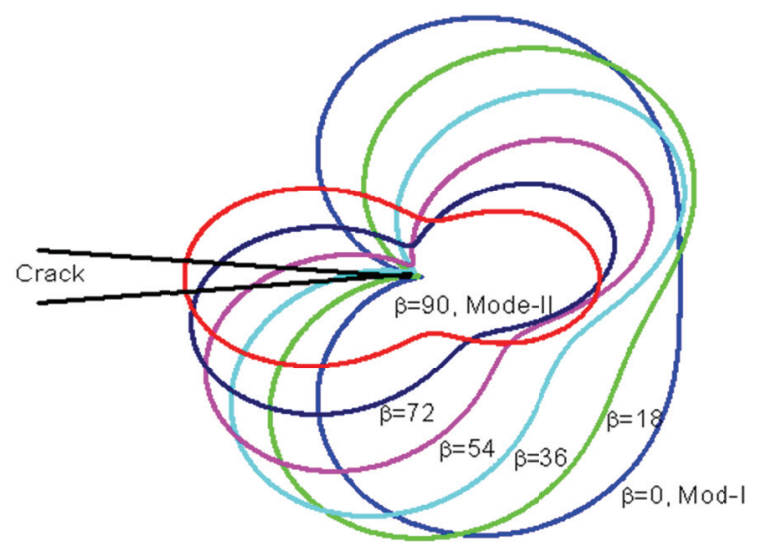

Figure 7: The sequential development of plastic zone obtained by analytical Eq. (10) for various loading angles.

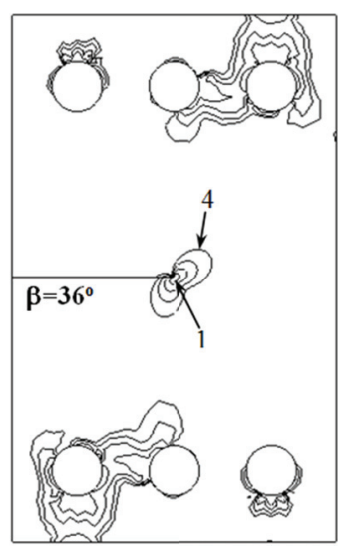

Figure 8: Sequential development of plastic zone for various applied loads. Number 1,2,3,4 indicates the plastic zone for applied load 4, 6, 8 and $10 \mathrm{kN}$. 
To illustrate the development of the crack-tip plastic zones for various loading angles, $\beta=0^{\circ}$ to $90^{\circ}$ and applied load $10 \mathrm{kN}$, the contours are superimposed and shown in Fig. 9. From this figure one can find that for similar applied load at various loading angles $(\beta)$, the plastic zone under-goes a rotation. It is also interesting to note that the shape of the plastic zone at various $\beta$ remains almost similar, with some change in the size and orientation. The nature of growth of plastic zone for various loading angles is in good agreement with the theoretical results as shown in Fig. 7 . From the results for plastic enclaves shown in Fig. 7, Fig. 8 and Fig. 9, several plastic-zone characterizing parameters can be estimated at various load steps and loading angle $(\beta)$, such as: (i) plastic zone size along the crack plane, $r_{p}$, (ii) maximum plastic zone size, $\left(r_{p}\right.$ )max, (iii) angle at which the maximum extent of plastic zone occurs, $\theta$, measured from the crack plane, (iv) minimum plastic zone radius (MPZR) and (v) angle at which MPZR occurs, $\theta_{\mathrm{o}}$. These parameters are schematically illustrated in Fig. 10.

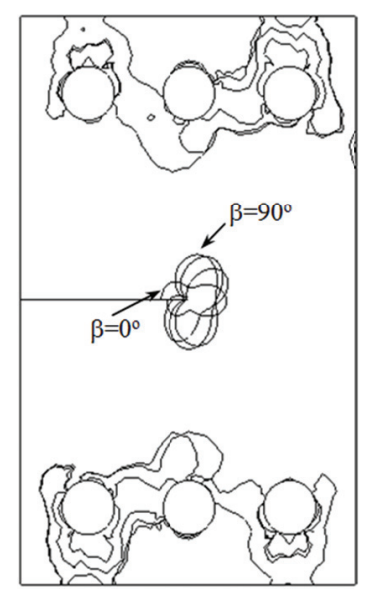

Figure 9: Typical shapes of crack-tip plastic zones for $\beta=0^{\circ}-90^{\circ}$ under $10 \mathrm{kN}$ load.

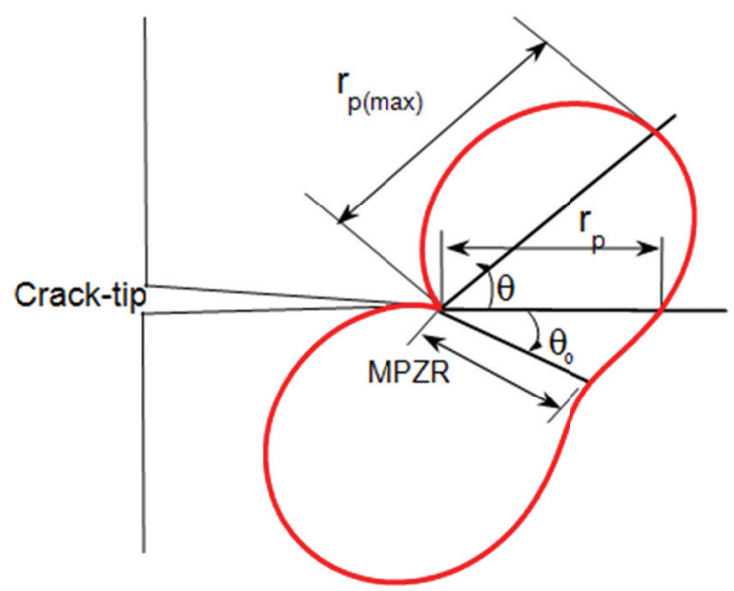

Figure 10: Schematic representation of plastic zone characterizing parameters.

The variation of the plastic zone characterizing parameters $r_{p}$ and $\left(r_{p}\right)_{\max } v s . K_{\text {eff }}$ and $\theta v s$. $\beta$ for CTS specimen is shown in Fig.11, Fig.12 and Fig.13 respectively. Fig. 11 shows the variation of $r_{p} v$ s. $K_{\text {eff }}$ for various loading angles $(\beta)$. The magnitudes of $r_{p}$ estimated by analytical Eq. 10 and FEA are compared for particular values of effective stress intensity factors. It must be noted that for various loading angles $(\beta)$, the plastic zone size along the crack plane for the finite element analysis is slightly larger than that of the theoretical values. It is found that there is less than $2 \%$ of error in estimation of both the analytical and FEA results. This discrepancy may be due to the finite element analysis approximation. Fig. 11 illustrates that the plastic zone size ahead of the crack-tip increases with $K_{\text {eff. }} I t$ is also clear from this figure that, for a particular magnitude of $K_{\text {eff }}$ (for example, $500 \mathrm{MPa} \mathrm{mm}^{1 / 2}$ ) the value of $\mathrm{r}_{\mathrm{p}}$ is least for $\beta=90^{\circ}$ (mode- $\mathrm{I}$ ) and it is highest for $\beta=0^{\circ}$ (mode-II). The difference in magnitudes of $r_{p}$ for $\beta=0^{\circ}$ and $90^{\circ}$ for $K_{\text {eff }}=500$ is about $5 \mathrm{~mm}$, which is 3.33 times that of Mode-I. These results infer that, due to minimum plastic zone radius ahead of crack-tip for a particular value of $\mathrm{K}_{\text {eff }}$, mode-I loading can lead to material fracture earlier than any mixed mode or mode-II loading.

The variation of the analytical and the finite element values of $\left(r_{p}\right)_{\max } v s$. $K_{\text {eff }}$ for various loading angles $(\beta)$ is depicted in Fig. 12. It is also noted that for various loading angles $(\beta)$, the maximum plastic zone size $\left(\mathrm{r}_{\mathrm{p}}\right)_{\max }$ estimated by finite element analysis is slightly larger than that of the analytical values for a particular effective stress intensity factor. It is also found that there is less than $2 \%$ of error in estimation of both the analytical and finite element results. This figure also indicates that the magnitude of $\left(r_{p}\right)_{\max }$ for a particular value of $K_{\text {eff }}$ is least for $\beta=90^{\circ}$ (mode-I) and it is highest for $\beta=0$ o (mode-II).

These results $\left(r_{p}\right.$ and $\left.\left(r_{p}\right)_{\max }\right)$ indicate clearly that the area of plastic zone in mode-I is much smaller than that of mode-II for the similar magnitude of $\mathrm{K}_{\text {eff. }}$ This analysis infers that for the similar magnitude of $\mathrm{K}_{\text {eff }}$ the energy absorption capacity of the material in Mode-I is much lower than under Mode-II loading. One can conclude from this analysis that, due to smaller plastic zone area ahead of the crack-tip, the mode-I loading leads to early fracture, hence in fracture, Mode-I loading is considered to be more dangerous than mode-II. The variation of the theoretical values of $\theta$ are compared with the FEA values of $\theta$ with the loading angle, $\beta$ is depicted in Fig. 13. A small discrepancy in the analytical and finite element values of $\theta$ with the loading angle, $\beta$ is observed. This plot indicates that the angle at which the maximum extent of plastic zone size occurs $(\theta)$ changes from $0^{\circ}$ to $90^{\circ}$ as $\beta$ is varied from $0^{\circ}$ to $90^{\circ}$ both for the analytical and the FEA results. It is 
interesting to find that $\beta=\theta$ for mode-I and mode-II loading conditions only. For the mixed mode $\left(\beta=36^{\circ}\right.$ to $\left.72^{\circ}\right)$ there is a considerable amount of deviation between $\beta$ and $\theta$ is observed. The nature of the variation of $\theta v s$. $\beta$ is almost similar for all various applied loads. From Fig. 13 one can understand that the extension of the plastic zone takes place almost in a similar angle $\theta$ for a constant loading angle, $\beta$.

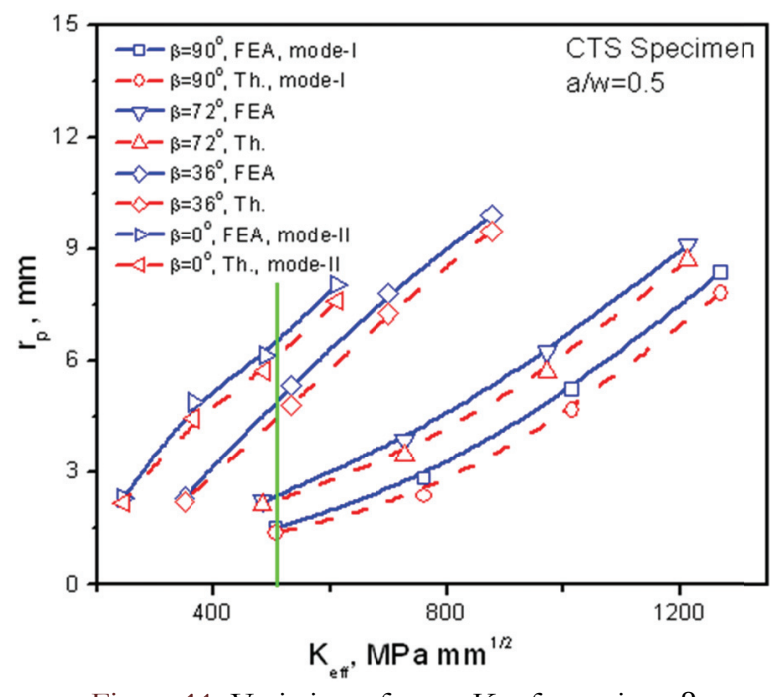

Figure 11: Variation of $\mathrm{r}_{\mathrm{p}} \mathrm{vs}$. $\mathrm{K}_{\mathrm{eff}}$ for various $\beta$

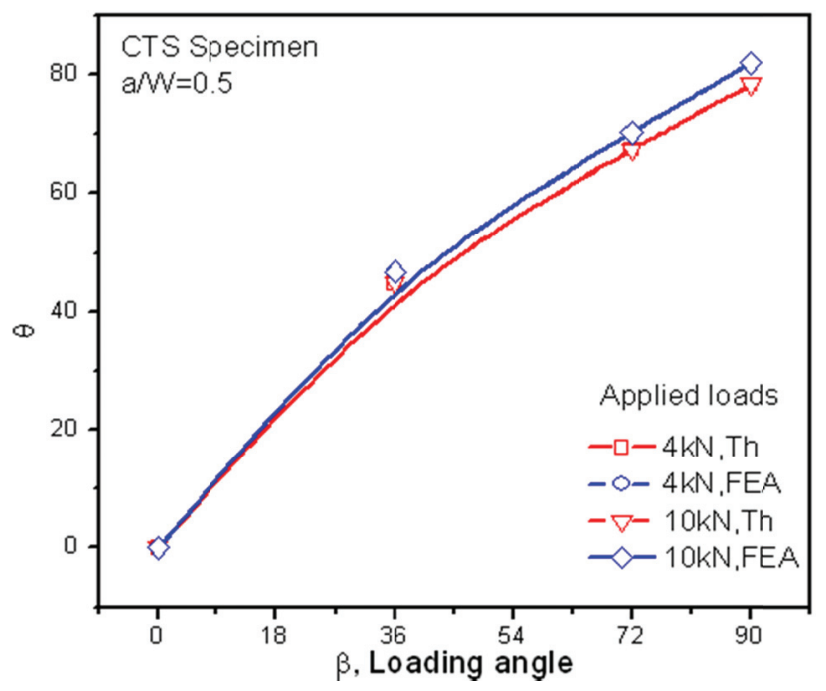

Figure 13: Variation of $\theta$ vs. $\beta$ for various loads

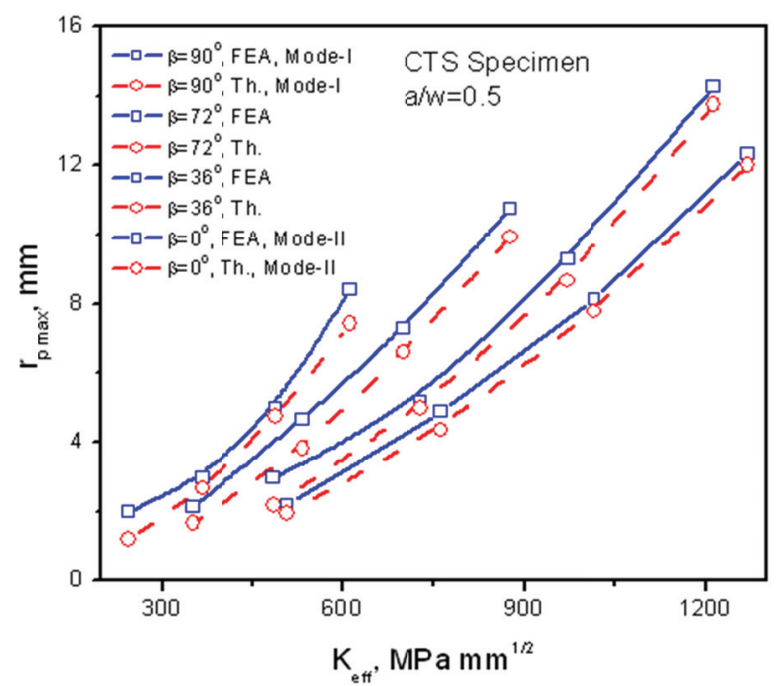

Figure 12: Variation of $\left(\mathrm{r}_{\mathrm{p}}\right)_{\max }$ vs. $\mathrm{K}_{\mathrm{eff}}$ for various $\beta$

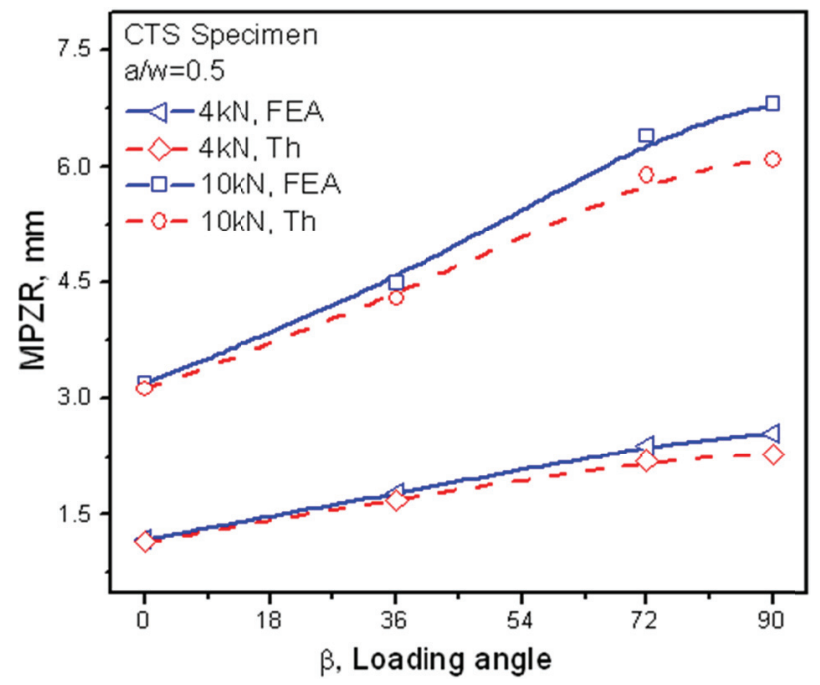

Figure 14: Variation of MPZR vs. $\beta$ for various loads

The variation of minimum plastic zone radius (MPZR) vs. loading angle, $\beta$, for various applied loads is depicted in Fig.14. The estimated theoretical values of minimum plastic zone radius have been superimposed with the FEA values of minimum plastic zone radius in Fig.14 typically for load $4 \mathrm{kN}$ and $10 \mathrm{kN}$. This plot indicates that there exists some discrepancy in the estimation of minimum plastic zone by analytical formulation and present finite element results. It is found that there is $10.5 \%$ and $2.4 \%$ error in estimation of minimum plastic zone for mode-I $\left(\beta=90^{\circ}\right)$ and mode-II $\left(\beta=0^{0}\right)$ loading angle respectively. The percentage error for the theoretical and FEA values of minimum plastic zone radius decreases from loading angle $90^{\circ}$ to $0^{\circ}$. This discrepancy in estimated magnitudes of MPZR attributed to approximation in the loading conditions in finite element analysis. This figure indicates that the magnitude of MPZR increases as the loading angle is changed from $0^{\circ}$ (Mode-II) to $90^{\circ}$ (Mode-I). It is interesting to note that the magnitude of MPZR in Mode-II loading is about 1.987 times less than that of Mode-I for applied load 4kN both for the analytical and finite element values. It is observed from Fig. 14 that the ratio of MPZR between Mode-I and Mode-II decreases with the increase in the applied load. In the case of applied load of $10 \mathrm{kN}$, the ratio is found to be 2.0 almost same as that of 
applied load $4 \mathrm{kN}$. These results clearly demonstrate that the specimen experiences minimum plastic zone radius under Mode-II loading only. The results of MPZR estimated using the theoretical and the finite element in this investigation can be used as inputs for minimum plastic zone radius (MPZR) criterion for crack initiation [4] in mixed mode loading for a CTS specimen.

The variation of the minimum plastic zone radius (MPZR) vs. effective stress intensity factor (Keff) is also studied. The plot of MPZR vs. $K_{\text {eff }}$ for various loading angles estimated by analytical and FEA are depicted in Fig.15a and Fig.15b respectively. It is interesting to note from these figures that the variation of of MPZR vs. $K_{\text {eff }}$ is linear and is independent of loading angles. These figures infer that for any loading angle (for the analytical and the FEA), the growth of MPZR is proportional to the effective stress intensity factor. The proportionality constant can be evaluated by fitting a straight line equation to all the MPZR data. Such a linear fit is shown in Fig.15a and Fig.15b. The slope of the estimated linear fit line is 0.0066 for analytical results and 0.0068 for FEA results. From these results, the relation between the MPZR and $K_{\text {eff }}$ independent of loading angle can be expressed as follows:

$$
\begin{aligned}
& \frac{M P Z R}{K_{e f f}}=0.0066 \frac{\mathrm{mm}^{\frac{1}{2}}}{M P a} \quad \text { (Analytical) } \\
& \frac{M P Z R}{K_{e f f}}=0.0068 \frac{\mathrm{mm}^{\frac{1}{2}}}{M P a}
\end{aligned}
$$

The Eqns. 15 and 16 show that there is an insignificant difference in the results obtained by FEA and analytical methods.
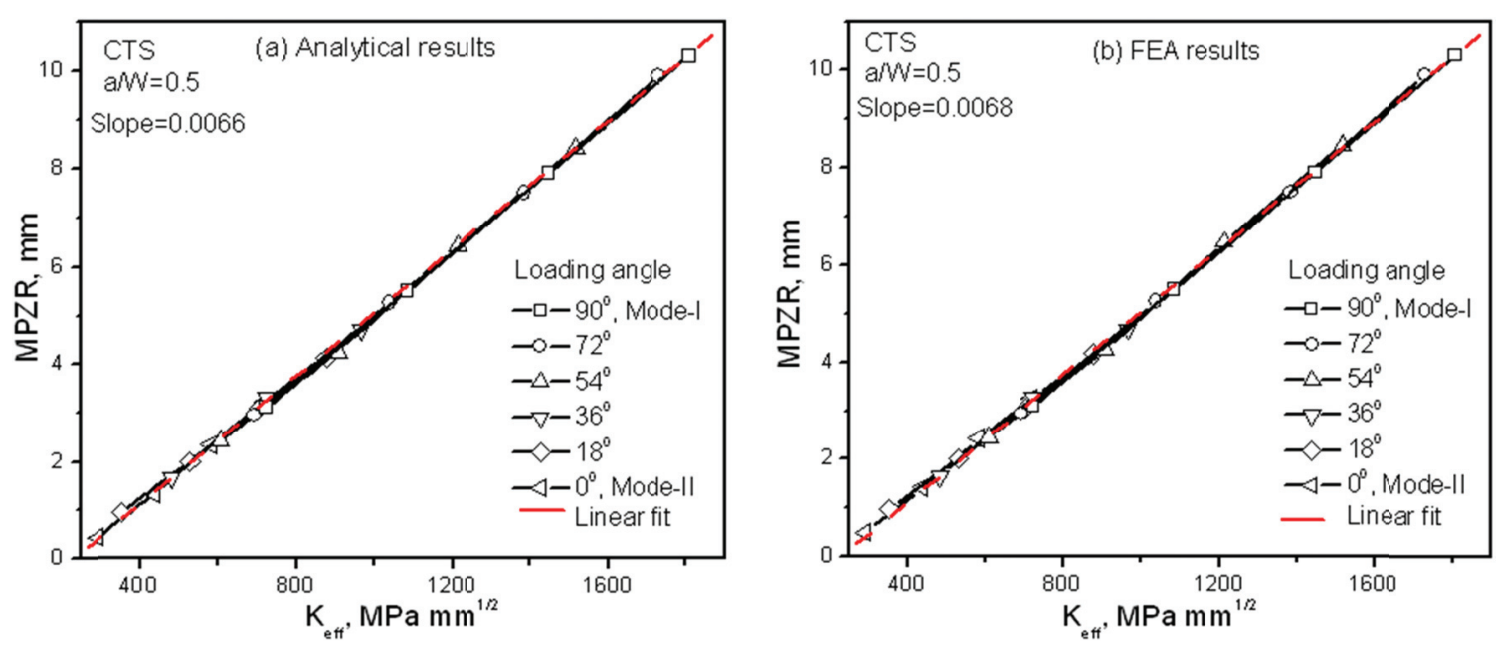

Figure 15: Variation of MPZR vs. $K_{\text {eff }}$ for various $\beta$ (a) analytical and (b) FEA results.

The Eqns. 15 and 16 can be used to estimate MPZR in a CTS specimen independent of the loading angle if $\mathrm{K}_{\text {eff }}$ is known, or vice versa. The proposed Eqns. 15 and 16 can be of great use in MPZR criteria.

In this investigation the angle at which MPZR occurs $\left(\theta_{0}\right)$ is also studied with respect to the loading angle $(\beta)$ both for the analytical and the finite element analysis. The variation of $\theta_{\mathrm{o}} v$ s. loading angle obtained in this analysis for various applied loads along with the similar results by Bian and Kim [4] is depicted in Fig.16. This figure shows that the magnitude of $\theta_{0}$ decreases from $82.5^{\circ}$ to $0^{\circ}$ as $\beta$ changes from $0^{\circ}$ to $90^{\circ}$. The results shown in Fig. 16 indicate that the magnitude of $\theta_{\mathrm{o}}$ computed for various applied loads and a particular $\beta$ is almost similar. A small dissimilarity observed in the estimated analytical $\theta_{\circ}$ compared with the FEA $\theta_{\circ}$ for various $\beta$ can possibly be attributed to varied loading condition in FEA through loading jig. Bian and Kim [4] have considered that the crack in mixed mode loading initiates in the direction of MPZR in a CTS specimen. These investigators have used the magnitude of $\theta_{0}$ for defining the crack initiation angle. The nature of the variation of $\theta_{\circ} v$ s. $\beta$ obtained in present analysis shown in Fig. 16 slightly differs from the theoretical results of Bian and Kim [4], as the theoretical results in [4] are obtained for plane strain analysis. Fig. 16 shows that for a specimen under Mode-I loading crack initiates along the ligament, and for a specimen under mode-II loading crack initiates almost perpendicular to the ligament. In this investigation a validation of minimum plastic zone radius (MPZR) 
criteria and crack initiation angle $\left(\theta_{0}\right)$ is made by considering a detail comparison of the analytical and the FEA for cracktip plastic zones and its various parameters under mixed mode loading.

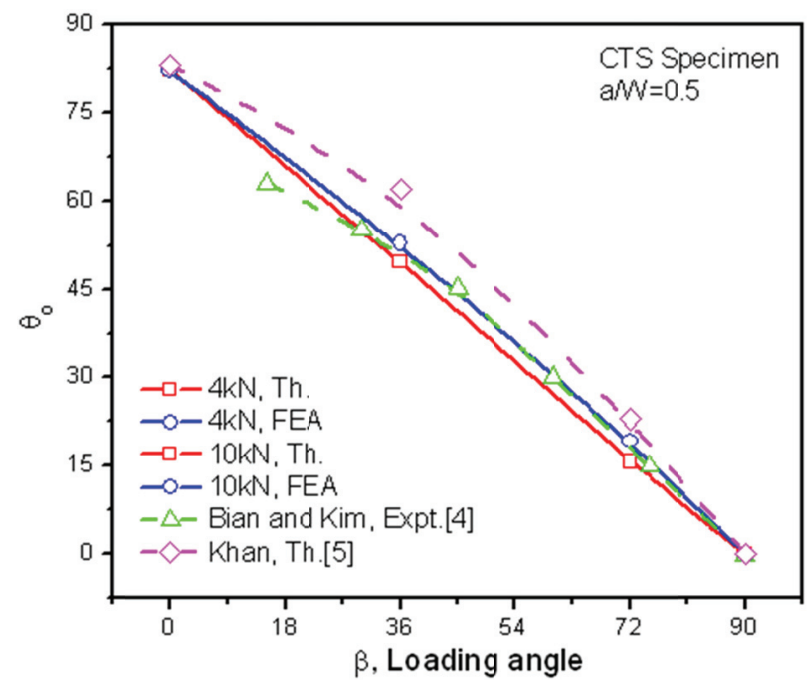

Figure 16: Variation of $\theta_{\mathrm{o}} v s . \beta$ for various loads.

\section{CONCLUSIONS}

he analytical results are in excellent agreement with the FEA results for CTS specimen under LEFM conditions. For the mixed mode analysis of CTS specimen one can use analytical formulations rather than going for extensive Finite element analysis.

\section{ACKNOWLEDGMENT}

uthors gratefully acknowledge the computational facilities provided by the Research Centre, B V B College of Engineering \& Technology, Hubli-580 031, India.

\section{REFERENCES}

[1] L. Nobile, Theoretical and Applied Fracture Mechanics, 33 (2000) 107.

[2] C. M. Sonsino, International Journal of Fatigue, 23 (2001) 159.

[3] X. Pitoiset, I. Rychlik, and A. Preumont, Fatigue and Fracture of Engineering Materials and Structures, 24 (2001) 715.

[4] L. C. Bian, K. S. Kim, International Journal of Fatigue, 26 (2004) 1169.

[5] K. Golos, B. Wasiluk, International Journal of Fracture, 102 (2000) 341.

[6] B. Wasiluk, K. Golos, Fatigue and Fracture of Engineering Materials and Structures, 23 (2000) 381.

[7] S. M. A. Kahan, M. K. Khraisheh, International Journal of Plasticity, 20 (2004) 55.

[8] S. K. Kudari, B. Maiti, K. K. Ray, Journal of Strain Analysis, 42 (2007) 125.

[9] K. H. Benrahou, M. Benguediab, M. Belhouri, Nait-Abdelaziz, A. Imad, Computational Materials Science, 38 (2007) 595.

[10] C. M. Sharanaprabhu, S. K. Kudari, American Institute of Physics, USA Editor: Professor Alexander M. Korsunsky, Oxford, England, (2009) 13.

[11] HA. Richard, Bruchvorhersagen bei überlagerter normal- und schubbeanspruchung von risen VDI Forschungsheft. Düsseldorf: VDI-Verlag, 631(1985) 1.

[12] L. P. Borrego, F. V. Antunes, J. M. Costa, J. M. Ferreira, International Journal of Fatigue, 28 (2006) 618.

[13] S. K. Kudari, C. M. Sharanaprabhu, International Journal of Engineering, Science and Technology, 2 (2010) 13.

[14] E. Gdoutos, G. Papakalitakis, International Journal of Fracture, 32 (1987) 143. 